\title{
THE JOINT UNIVERSALITY AND THE FUNCTIONAL INDEPENDENCE FOR LERCH ZETA-FUNCTIONS
}

\author{
ANTANAS LAURINČIKAS ${ }^{1}$ AND KOHJI MATSUMOTO
}

\begin{abstract}
The joint universality theorem for Lerch zeta-functions $L\left(\lambda_{l}, \alpha_{l}, s\right)$ $(1 \leq l \leq n)$ is proved, in the case when $\lambda_{l}$ s are rational numbers and $\alpha_{l}$ s are transcendental numbers. The case $n=1$ was known before ([12]); the rationality of $\lambda_{l}$ s is used to establish the theorem for the "joint" case $n \geq 2$. As a corollary, the joint functional independence for those functions is shown.
\end{abstract}

\section{Introduction}

Let $s=\sigma+i t$ be a complex variable, and let $\mathbb{N}, \mathbb{Z}, \mathbb{Q}, \mathbb{R}$ and $\mathbb{C}$ denote the set of all natural numbers, integers, rational numbers, real numbers and complex numbers, respectively. The Lerch zeta-function $L(\lambda, \alpha, s)$, for $\sigma>1$, is defined by

$$
L(\lambda, \alpha, s)=\sum_{m=0}^{\infty} \frac{e^{2 \pi i \lambda m}}{(m+\alpha)^{s}}
$$

Here $\alpha, \lambda \in \mathbb{R}, 0<\alpha \leq 1$, are fixed parameters. When $\lambda \in \mathbb{Z}$ the Lerch zetafunction $L(\lambda, \alpha, s)$ reduces to the Hurwitz zeta-function $\zeta(s, \alpha)$. If $\lambda \notin \mathbb{Z}$, then the function $L(\lambda, \alpha, s)$ is analytically continuable to an entire function. Clearly, in this case we may suppose that $0<\lambda<1$. In what follows we will deal with this case only.

The Lerch zeta-function is one of the classical objects in number theory, introduced by M. Lerch [16] in 1887.

In recent years the value-distribution of the Lerch zeta-function was studied by D. Klusch, R. Garunkštis, M. Katsurada, W. Zhang, by the authors and other mathematicians. In [12] the universality theorem for the function $L(\lambda, \alpha, s)$ was proved. In order to state it we need some notation.

Received February 18, 1999.

${ }^{1}$ Partially supported by Grant from Lithuanian Foundation of Studies and Science. 
By meas $\{A\}$ we denote the Lebesgue measure of the set $A$, and, for $T>0$, we use the notation

$$
\nu_{T}(\ldots)=\frac{1}{T} \operatorname{meas}\{\tau \in[0, T], \ldots\},
$$

where in place of dots some condition satisfied by $\tau$ is to be written. Let $D=\{s \in \mathbb{C}: 1 / 2<\sigma<1\}$. Then the result of [12] is as follows.

Let $\alpha$ be a transcendental number. Let $K$ be a compact subset of the strip $D$ with the connected complement, $f(s)$ be a continuous function on $K$ which is analytic in the interior of $K$. Then for any $\varepsilon>0$ it holds that

$$
\liminf _{T \rightarrow \infty} \nu_{T}\left(\sup _{s \in K}|L(\lambda, \alpha, s+i \tau)-f(s)|<\varepsilon\right)>0 .
$$

The universality for $L(\lambda, \alpha, s)$ was also studied in [5], [13].

It is the purpose of the present paper to obtain a joint universality theorem for Lerch zeta-functions. Suppose $n \geq 2$.

THEOREM 1. Let $\alpha_{1}, \ldots, \alpha_{n}$ be transcendental numbers, $\lambda_{1}=a_{1} / q_{1}$, $\ldots, \lambda_{n}=a_{n} / q_{n},\left(a_{1}, q_{1}\right)=1, \ldots,\left(a_{n}, q_{n}\right)=1$, where $q_{1}, \ldots, q_{n}$ are distinct positive integers and $a_{1}, \ldots, a_{n}$ are positive integers with $a_{1}<q_{1}, \ldots, a_{n}<$ $q_{n}$. Let $K_{1}, \ldots, K_{n}$ be compact subsets of the strip $D$ with connected complements, and, for $1 \leq l \leq n$, let $f_{l}(s)$ be a continuous function on $K_{l}$ which is analytic in the interior of $K_{l}$. Then for every $\varepsilon>0$ it holds that

$$
\liminf _{T \rightarrow \infty} \nu_{T}\left(\sup _{1 \leq l \leq n} \sup _{s \in K_{l}}\left|L\left(\lambda_{l}, \alpha_{l}, s+i \tau\right)-f_{l}(s)\right|<\varepsilon\right)>0 .
$$

Joint universality theorems for Dirichlet $L$-functions were obtained by B. Bagchi [2], [3], S.M. Gonek [7], and S.M. Voronin [18], [19]. For more general Dirichlet series such theorems were proved in [8], [9], [14].

The proof of Theorem 1 is based on Bagchi's method [2], [3], but some new ideas are necessary for the proof of Lemmas 5 and 6 below.

In the case of the aforementioned universality theorem [12] for a single zeta-function, the arithmetic nature of $\lambda$ is irrelevant. However, in the proof of Theorem 1 , the fact that $\lambda_{l} \in \mathbb{Q}(1 \leq l \leq n)$ is used essentially. In Section 4 we will discuss briefly the case when $\lambda_{l} \notin \mathbb{Q}$.

As an application of Theorem 1, we will show the joint functional independence. 
TheOrem 2. Let $\alpha_{l}, \lambda_{l}=a_{l} / q_{l}$ be as in Theorem 1 , and $F_{j}(0 \leq j \leq k)$ be continuous functions on $\mathbb{C}^{N n}$. Suppose

$$
\begin{aligned}
& \sum_{j=0}^{k} s^{j} F_{j}\left(L\left(\lambda_{1}, \alpha_{1}, s\right), \ldots, L\left(\lambda_{n}, \alpha_{n}, s\right), L^{\prime}\left(\lambda_{1}, \alpha_{1}, s\right), \ldots\right. \\
&\left.L^{\prime}\left(\lambda_{n}, \alpha_{n}, s\right), \ldots, L^{(N-1)}\left(\lambda_{1}, \alpha_{1}, s\right), \ldots, L^{(N-1)}\left(\lambda_{n}, \alpha_{n}, s\right)\right)=0
\end{aligned}
$$

identically for all $s \in \mathbb{C}$. Then $F_{j} \equiv 0(0 \leq j \leq k)$.

This theorem gives a generalization of the result proved in GarunkštisLaurinčikas [6]. A quite different approach to this type of problems has recently been developed by Amou-Katsurada [1].

\section{A joint limit theorem for Lerch zeta-functions}

For the proof of Theorem 1 we will apply a joint limit theorem in the sense of weak convergence of probability measures for the Lerch zetafunctions $L\left(\lambda_{1}, \alpha_{1}, s\right), \ldots L\left(\lambda_{n}, \alpha_{n}, s\right)$ in the space of analytic functions. Denote by $H(D)$ the space of analytic on $D$ functions equipped with the topology of uniform convergence on compacta. Let $\mathcal{B}(S)$ stand for the class of Borel sets of the space $S$. Define on $\left(H^{n}(D), \mathcal{B}\left(H^{n}(D)\right)\right)$ the probability measure

$P_{T}(A)=\nu_{T}\left(\left(L\left(\lambda_{1}, \alpha_{1}, s+i \tau\right), \ldots, L\left(\lambda_{n}, \alpha_{n}, s+i \tau\right)\right) \in A\right), \quad A \in \mathcal{B}\left(H^{n}(D)\right)$.

What we need is a limit theorem in the sense of weak convergence of probability measures for $P_{T}$ as $T \rightarrow \infty$, with an explicit form of the limit measure. Denote by $\gamma$ the unit circle on $\mathbb{C}$, i.e. $\gamma=\{s \in \mathbb{C}:|s|=1\}$, and let

$$
\Omega=\prod_{m=0}^{\infty} \gamma_{m},
$$

where $\gamma_{m}=\gamma$ for all $m=0,1,2, \ldots$ With the product topology and pointwise multiplication the infinite dimensional torus $\Omega$ is a compact topological Abelian group. Denoting by $m_{H}$ the probability Haar measure on $(\Omega, \mathcal{B}(\Omega))$, we obtain the probability space $\left(\Omega, \mathcal{B}(\Omega), m_{H}\right)$. Let $\omega(m)$ be the projection of $\omega \in \Omega$ to the coordinate space $\gamma_{m}$, and define on the probability space $\left(\Omega, \mathcal{B}(\Omega), m_{H}\right)$ the $H^{n}(D)$-valued random element $L(s, \omega)$ by

$$
L(s, \omega)=\left(L\left(\lambda_{1}, \alpha_{1}, s, \omega\right), \ldots, L\left(\lambda_{n}, \alpha_{n}, s, \omega\right)\right),
$$


where

$$
L\left(\lambda_{l}, \alpha_{l}, s, \omega\right)=\sum_{m=0}^{\infty} \frac{e^{2 \pi i \lambda_{l} m} \omega(m)}{\left(m+\alpha_{l}\right)^{s}}, \quad s \in D, \omega \in \Omega, l=1, \ldots, n
$$

The proof that $L\left(\lambda_{l}, \alpha_{l}, s, \omega\right)$ is an $H(D)$-valued random element can be found in [11]. Let $P_{L}$ stand for the distribution of the random element $L(s, \omega)$, i.e.

$$
P_{L}(A)=m_{H}(\omega \in \Omega: L(s, \omega) \in A), \quad A \in \mathcal{B}\left(H^{n}(D)\right)
$$

Lemma 1. The probability measure $P_{T}$ converges weakly to $P_{L}$ as $T \rightarrow$ $\infty$.

Proof. Let $D_{0}=\{s \in \mathbb{C}: \sigma>1 / 2\}$. Then in [15] the result of the lemma was proved in the case of the space $H^{n}\left(D_{0}\right)$. Obviously, from this the lemma follows.

\section{The support of the random element $L$}

In this section we will consider the support of the measure $P_{L}$. We recall that the minimal closed set $S_{P_{L}} \subseteq H^{n}(D)$ such that $P_{L}\left(S_{P_{L}}\right)=1$ is called the support of $P_{L}$. The set $S_{P_{L}}$ consists of all $f \in H^{n}(D)$ such that for every neighbourhood $\mathcal{G}$ of $f$ the inequality $P_{L}(\mathcal{G})>0$ is satisfied.

The support of the distribution of the random element $X$ is called the support of $X$ and is denoted by $S_{X}$.

LEMma 2. Let $\left\{X_{m}\right\}$ be a sequence of independent $H^{n}(D)$-valued random elements, and suppose that the series

$$
\sum_{m=1}^{\infty} X_{m}
$$

converges almost surely. Then the support of the sum of this series is the closure of the set of all $\underline{f} \in H^{n}(D)$ which may be written as a convergent series

$$
\underline{f}=\sum_{m=1}^{\infty} \underline{f}_{m}, \quad \underline{f}_{m} \in S_{X_{m}}
$$


Proof of the lemma in the case $n=1$ is given in [10], Theorem 1.7.10. The proof when $n>1$ is similar to that of the case $n=1$.

Let $\underline{f}(s)=\left(f_{1}(s), \ldots, f_{n}(s)\right) \in H^{n}(D)$. Then we write

$$
|\underline{f}(s)|^{2}=\sum_{l=1}^{n}\left|f_{l}(s)\right|^{2} .
$$

Lemma 3. Let $\left\{\underline{f}_{m}=\left(f_{1 m}, \ldots, f_{n m}\right), m \geq 1\right\}$ be a sequence in $H^{n}(D)$ which satisfies:

a) If $\mu_{1}, \ldots, \mu_{n}$ are complex measures on $(\mathbb{C}, \mathcal{B}(\mathbb{C}))$ with compact supports contained in $D$ such that

$$
\sum_{m=1}^{\infty}\left|\sum_{l=1}^{n} \int_{\mathbb{C}} f_{l m} d \mu_{l}\right|<\infty
$$

then

$$
\int_{\mathbb{C}} s^{r} d \mu_{l}(s)=0
$$

for all $l=1, \ldots, n, r=0,1,2, \ldots$.

b) The series

$$
\sum_{m=1}^{\infty} \underline{f}_{m}
$$

converges in $H^{n}(D)$.

c) For any compact $K \subseteq D$

$$
\sum_{m=1}^{\infty} \sup _{s \in K}\left|\underline{f}_{m}(s)\right|^{2}<\infty .
$$

Then the set of all convergent series

$$
\sum_{m=1}^{\infty} a_{m} \underline{f}_{m}
$$

with $a_{m} \in \gamma$ is dense in $H^{n}(D)$.

Proof. This lemma is Lemma 5.2.9 of [2], see also [3]. In [10] the proof in the case $n=1$ is given, see Theorem 6.3.10. The proof of the general case is obtained in a similar way. 
Now we state two lemmas on entire functions of exponential type. Recall that an entire function $f(s)$ is of exponential type if

$$
\limsup _{r \rightarrow \infty} \frac{\log \left|f\left(r e^{i \theta}\right)\right|}{r}<\infty
$$

uniformly in $\theta,|\theta| \leq \pi$.

Lemma 4. Let $\mu$ be a complex measure on $(\mathbb{C}, \mathcal{B}(\mathbb{C}))$ with the compact support contained in the half-plane $\sigma>\sigma_{0}$, and let

$$
f(z)=\int_{\mathbb{C}} e^{s z} d \mu(s), \quad z \in \mathbb{C} .
$$

If $f(z) \not \equiv 0$, then

$$
\limsup _{x \rightarrow \infty} \frac{\log |f(x)|}{x}>\sigma_{0} .
$$

This lemma is due to B. Bagchi [2]. For the proof see Lemma 6.4.10 of $[10]$.

Let $\mathcal{M}$ be a set of natural numbers having a positive density, i.e.

$$
\lim _{x \rightarrow \infty} \frac{1}{x} \#\{m \in \mathcal{M}: m \leq x\}=d>0 .
$$

LEMMA 5. Let $f(s)$ be an entire function of exponential type, and let

$$
\limsup _{r \rightarrow \infty} \frac{\log |f(r)|}{r}>-1 \text {. }
$$

Then

$$
\sum_{m \in \mathcal{M}}|f(\log m)|=\infty
$$

Proof. Let $\alpha>0$ be such that

$$
\limsup _{y \rightarrow \infty} \frac{\log |f( \pm i y)|}{y} \leq \alpha .
$$

Let us fix a positive number $\beta$ such that $\alpha \beta<\pi$, and suppose, on the contrary, that

$$
\sum_{m \in \mathcal{M}}|f(\log m)|<\infty
$$


Consider the set $A=\{m \in \mathbb{N}: \exists r \in((m-1 / 4) \beta,(m+1 / 4) \beta]$ and $|f(r)| \leq$ $\left.e^{-r}\right\}$. Let, for brevity,

$$
m_{\mathcal{M}}(x)=\sum_{\substack{m \leq x \\ m \in \mathcal{M}}} 1
$$

Clearly, we have

$$
\sum_{m \in \mathcal{M}}|f(\log m)| \geq \sum_{m \notin A} \sum_{m}^{\prime}|f(\log k)| \geq \sum_{m \notin A} \sum_{m}^{\prime} \frac{1}{k},
$$

where $\sum_{m}^{\prime}$ denotes the sum extended over all natural numbers $k \in \mathcal{M}$ satisfying $(m-1 / 4) \beta<\log k \leq(m+1 / 4) \beta$. If we denote

$$
a=\exp \left\{\left(m-\frac{1}{4}\right) \beta\right\}, \quad b=\exp \left\{\left(m+\frac{1}{4}\right) \beta\right\},
$$

then we have that

$$
\sum_{m}^{\prime} \frac{1}{k}=\sum_{\substack{k \in \mathcal{M} \\ a<k \leq b}} \frac{1}{k}
$$

Summing by parts, we find

$$
\sum_{\substack{k \in \mathcal{M} \\ a<k \leq b}} \frac{1}{k}=\frac{1}{b} \sum_{\substack{k \in \mathcal{M} \\ a<k \leq b}} 1+\int_{a}^{b}\left(\sum_{\substack{k \in \mathcal{M} \\ a<k \leq u}} 1\right) \frac{d u}{u^{2}}
$$

Obviously,

$$
\sum_{\substack{k \in \mathcal{M} \\ a<k \leq u}} 1=m_{\mathcal{M}}(u)-m_{\mathcal{M}}(a)
$$

The assumption (1) implies

$$
m_{\mathcal{M}}(x)=d x(1+o(1)), \quad x \rightarrow \infty,
$$

hence, for any $\varepsilon>0$, there exists a number $x_{0}=x_{0}(\varepsilon)$ such that

$$
\begin{aligned}
& m_{\mathcal{M}}(u) \geq d u(1-\varepsilon), \\
& m_{\mathcal{M}}(a) \leq d a(1+\varepsilon)
\end{aligned}
$$

if $a \geq x_{0}$. Therefore

$$
\sum_{\substack{k \in \mathcal{M} \\ a<k \leq u}} 1 \geq d((u-a)-\varepsilon(a+u))
$$


Let $\eta$ satisfy the inequality $1<\eta<\exp \{\beta / 2\}$, and consider the case $u \geq \eta a$. Then we have

$$
\begin{aligned}
\frac{1}{2}(u-a)-\varepsilon(a+u) & \geq \frac{1}{2}\left(u-\frac{u}{\eta}\right)-\varepsilon\left(\frac{u}{\eta}+u\right) \\
& =u\left(\left(\frac{1}{2}-\varepsilon\right)-\frac{1}{\eta}\left(\frac{1}{2}+\varepsilon\right)\right)>0
\end{aligned}
$$

if we choose $\varepsilon$ sufficiently small. Hence and from (6) we obtain

$$
\sum_{\substack{k \in \mathcal{M} \\ a<k \leq u}} 1 \geq \frac{d}{2}(u-a), \quad u \geq \eta a .
$$

Combining this with (5), and using partial summation again, we have

$$
\text { (7) } \begin{aligned}
\sum_{\substack{k \in \mathcal{M} \\
a<k \leq b}} \frac{1}{k} & \geq \frac{d}{2 b}(b-a)+\frac{d}{2} \int_{\eta a}^{b}(u-a) \frac{d u}{u^{2}} \\
& \geq \frac{d}{2}\left\{\frac{1}{b}([b]-[\eta a])+\int_{\eta a}^{b}([u]-[\eta a]) \frac{d u}{u^{2}}+\frac{B}{a}\right\}=\frac{d}{2} \sum_{\eta a<k \leq b} \frac{1}{k}+\frac{B}{a}
\end{aligned}
$$

where $[x]$ denotes the integer part of $x$, and $B$ is a number (not always the same) bounded by a constant. Clearly,

$$
\begin{aligned}
\sum_{\eta a<k \leq b} \frac{1}{k} & =\log b-\log (\eta a)+\frac{B}{\eta a} \\
& =\left(m+\frac{1}{4}\right) \beta-\log \eta-\left(m-\frac{1}{4}\right) \beta+B e^{-m \beta} \\
& =\frac{\beta}{2}-\log \eta+B e^{-m \beta}
\end{aligned}
$$

From the choice of $\eta$ it follows that

$$
\frac{\beta}{2}-\log \eta>0 \text {. }
$$

Now (7) shows

$$
\sum_{\substack{k \in \mathcal{M} \\ a<k \leq b}} \frac{1}{k} \geq \frac{d}{2}\left(\frac{\beta}{2}-\log \eta\right)+B e^{-m \beta}
$$


This together with (3) and (4) implies

$$
\sum_{m \notin A}\left(\frac{d}{2}\left(\frac{\beta}{2}-\log \eta\right)+B e^{-m \beta}\right) \leq \sum_{m \in \mathcal{M}}|f(\log m)|<\infty
$$

hence

$$
\sum_{m \notin A} 1<\infty
$$

Let $A=\left\{a_{m}: a_{1}<a_{2}<\ldots\right\}$. Then (8) gives that

$$
\lim _{m \rightarrow \infty} \frac{a_{m}}{m}=1 \text {. }
$$

By the definition of the set $A$, there exists a sequence $\left\{\lambda_{m}\right\}$ such that

$$
\left(a_{m}-\frac{1}{4}\right) \beta<\lambda_{m} \leq\left(a_{m}+\frac{1}{4}\right) \beta,
$$

and $\left|f\left(\lambda_{m}\right)\right| \leq e^{-\lambda_{m}}$. Hence, in view of (9),

$$
\lim _{m \rightarrow \infty} \frac{\lambda_{m}}{m}=\beta,
$$

and

$$
\limsup _{m \rightarrow \infty} \frac{\log \left|f\left(\lambda_{m}\right)\right|}{\lambda_{m}} \leq-1 .
$$

Now we apply Theorem 6.4 .12 of [10]. The assumptions of that theorem are satisfied by (10), (2), and the condition $\alpha \beta<\pi$. Hence by that theorem it follows that

$$
\limsup _{r \rightarrow \infty} \frac{\log |f(r)|}{r} \leq-1 .
$$

This contradicts the assumption of the lemma, and Lemma 5 is proved.

Lemma 6. The support of the measure $P_{L}$ is the whole of $H^{n}(D)$.

Proof. It follows from the definition of $\Omega$ that $\{\omega(m)\}$ is a sequence of independent random variables with respect to the measure $m_{H}$. Hence $\left\{\underline{f}_{m}(s, \omega(m)), m \in \mathbb{N} \cup\{0\}\right\}$ is a sequence of independent $H^{n}(D)$-valued random elements, where

$$
\underline{f}_{m}(s, \omega(m))=\left(\frac{e^{2 \pi i \lambda_{1} m} \omega(m)}{\left(m+\alpha_{1}\right)^{s}}, \ldots, \frac{e^{2 \pi i \lambda_{n} m} \omega(m)}{\left(m+\alpha_{n}\right)^{s}}\right) .
$$


The support of each $\omega(m)$ is the unit circle $\gamma$. Therefore the set $\left\{\underline{f}_{m}(s, a)\right.$ : $a \in \gamma\}$ is the support of the random element $\underline{f}_{m}(s, \omega(m))$. Consequently, by Lemma 2 the closure of the set of all convergent series

$$
\sum_{m=0}^{\infty} \underline{f}_{m}\left(s, a_{m}\right), \quad a_{m} \in \gamma
$$

is the support of the random element $L(s, \omega)$. It remains to check that the latter set is dense in $H^{n}(D)$.

Let $\mu_{1}, \ldots, \mu_{n}$ be complex measures on $(\mathbb{C}, \mathcal{B}(\mathbb{C}))$ with compact supports contained in $D$ such that

$$
\sum_{m=0}^{\infty}\left|\sum_{l=1}^{n} \int_{\mathbb{C}} \frac{e^{2 \pi i \lambda_{l} m}}{\left(m+\alpha_{l}\right)^{s}} d \mu_{l}(s)\right|<\infty .
$$

It is well known that for all $s \in \mathbb{C}$

$$
e^{s}=1+B|s| e^{|s|} .
$$

Therefore, for $m \geq 2$,

$$
\begin{aligned}
\left(m+\alpha_{l}\right)^{-s} & =m^{-s}\left(1+\frac{\alpha_{l}}{m}\right)^{-s}=m^{-s} \exp \left\{-s \log \left(1+\frac{\alpha_{l}}{m}\right)\right\} \\
& =m^{-s} \exp \left\{\frac{B|s|}{m}\right\}=m^{-s}\left(1+\frac{B|s|}{m} e^{B|s|}\right) \\
& =m^{-s}+B m^{-1-\sigma}|s| e^{B|s|} .
\end{aligned}
$$

Hence, taking into account the properties of the measures $\mu_{1}, \ldots, \mu_{n}$, we deduce from (11) that

$$
\sum_{m=0}^{\infty}\left|\sum_{l=1}^{n} \int_{\mathbb{C}} \frac{e^{2 \pi i \lambda_{l} m}}{m^{s}} d \mu_{l}(s)\right|<\infty
$$

which can be rewritten in the form

$$
\sum_{\substack{m=0 \\ m \equiv r(\bmod q)}}^{\infty}\left|\sum_{l=1}^{n} \int_{\mathbb{C}} \frac{e^{2 \pi i \lambda_{l} r}}{m^{s}} d \mu_{l}(s)\right|<\infty, \quad 1 \leq r \leq q
$$

where $q=\left[q_{1}, \ldots, q_{n}\right]$. Now let

$$
\nu_{r}(A)=\sum_{l=1}^{n} e^{2 \pi i \lambda_{l} r} \mu_{l}(A), \quad A \in \mathcal{B}(\mathbb{C}), 1 \leq r \leq q
$$


Note that the measures $\nu_{1}, \ldots, \nu_{q}$ have the same properties as $\mu_{1}, \ldots, \mu_{n}$. Using this notation, we may write the relation (12) as follows:

$$
\sum_{\substack{m=0 \\ m \equiv r(\bmod q)}}^{\infty}\left|\int_{\mathbb{C}} m^{-s} d \nu_{r}(s)\right|<\infty, \quad 1 \leq r \leq q .
$$

Let

$$
\tilde{\varrho}_{r}(z)=\int_{\mathbb{C}} e^{-s z} d \nu_{r}(s), \quad z \in \mathbb{C} .
$$

Then (13) becomes the following condition

$$
\sum_{\substack{m=0 \\ m \equiv r(\bmod q)}}^{\infty}\left|\tilde{\varrho}_{r}(\log m)\right|<\infty, \quad 1 \leq r \leq q .
$$

By Lemma 4 we obtain that $\tilde{\varrho}_{r}(z) \equiv 0$, or

$$
\limsup _{x \rightarrow \infty} \frac{\log \left|\tilde{\varrho}_{r}(x)\right|}{x}>-1, \quad 1 \leq r \leq q .
$$

Lemma 5 shows that the last inequality contradicts (14). Hence

$$
\tilde{\varrho}_{r}(z) \equiv 0
$$

for $1 \leq r \leq q$. Let

$$
\varrho_{l}(z)=\int_{\mathbb{C}} e^{-s z} d \mu_{l}(s), \quad z \in \mathbb{C}, l=1, \ldots, n .
$$

Then by the definitions of $\nu_{r}$ and $\tilde{\varrho}_{r}$ we have

$$
\begin{aligned}
\tilde{\varrho}_{r}(z) & =\int_{\mathbb{C}} e^{-s z} \sum_{l=1}^{n} e^{2 \pi i \lambda_{l} r} d \mu_{l}(s)=\sum_{l=1}^{n} e^{2 \pi i \lambda_{l} r} \int_{\mathbb{C}} e^{-s z} d \mu_{l}(s) \\
& =\sum_{l=1}^{n} e^{2 \pi i \lambda_{l} r} \varrho_{l}(z),
\end{aligned}
$$

which is identically equal to zero by (15). Multiplying by $e^{-2 \pi i \lambda_{j}}$, we have

$$
\sum_{l=1}^{n} e^{2 \pi i\left(\lambda_{l}-\lambda_{j}\right) r} \varrho_{l}(z) \equiv 0, \quad 1 \leq r \leq q .
$$


Taking into account that

$$
\sum_{r=1}^{q} e^{2 \pi i\left(\lambda_{l}-\lambda_{j}\right) r}= \begin{cases}q & \text { if }\left(\lambda_{l}-\lambda_{j}\right) \in \mathbb{Z} \\ 0 & \text { otherwise }\end{cases}
$$

and the fact that $\lambda_{l}-\lambda_{j} \in \mathbb{Z}$ only if $l=j$, and summing (16) over $r=$ $1, \ldots, q$, we find that

$$
\varrho_{j}(z)=\int_{\mathbb{C}} e^{-s z} d \mu_{j}(s) \equiv 0, \quad j=1,2, \ldots, n .
$$

Differentiating this equality $r$ times and then putting $z=0$, we find that

$$
\int_{\mathbb{C}} s^{r} \mu_{j}(s)=0
$$

for all $j=1, \ldots, n, r=0,1,2, \ldots$. Thus the condition a) of Lemma 3 for the sequence $\left\{\underline{f}_{m}(s, 1), m \geq 1\right\}$ is satisfied.

Let, for a natural number $N$,

$$
S(\lambda, N)=\sum_{m=0}^{N} e^{2 \pi i \lambda m}
$$

If $\lambda \notin \mathbb{Z}$, then we have

$$
S(\lambda, N)=\frac{1-e^{2 \pi i \lambda(N+1)}}{1-e^{2 \pi i \lambda}}
$$

which is uniformly bounded for all $N \geq 1$. Summing by parts, we find

$$
\sum_{m=0}^{N} \frac{e^{2 \pi i \lambda m}}{(m+\alpha)^{s}}=S(\lambda, N)(N+\alpha)^{-s}+s \int_{0}^{N} S(\lambda, u) \frac{d u}{(u+\alpha)^{s+1}} .
$$

Taking $N \rightarrow \infty$ we obtain

$$
\sum_{m=0}^{\infty} \frac{e^{2 \pi i \lambda m}}{(m+\alpha)^{s}}=s \int_{0}^{\infty} S(\lambda, u) \frac{d u}{(u+\alpha)^{s+1}}
$$

which converges for $\sigma>0$ in view of (17). Consequently, the series

$$
\sum_{m=0}^{\infty} \frac{e^{2 \pi i \lambda m}}{(m+\alpha)^{s}}
$$


with $\lambda \notin \mathbb{Z}$ converges (Corollary 2.1.3 of [10]) uniformly on compacta in the half-plane $\sigma>\sigma_{0}$ for any $\sigma_{0}>0$. This shows that the series

$$
\sum_{m=0}^{\infty} \underline{f}_{m}(s, 1)
$$

converges in $H^{n}(D)$, i.e. the condition b) of Lemma 3 holds for the sequence $\left\{\underline{f}_{m}(s, 1), m \geq 1\right\}$. The condition c) of Lemma 3 is also satisfied clearly, since for $s \in K$ we have that $\sigma>1 / 2$.

Now, applying Lemma 3, we have that the set of all convergent series

$$
\sum_{m=0}^{\infty} a_{m} \underline{f}_{m}(s, 1)=\sum_{m=0}^{\infty} \underline{f}_{m}\left(s, a_{m}\right)
$$

with $a_{m} \in \gamma$ is dense in $H^{n}(D)$. This completes the proof of the lemma.

\section{Proof of Theorem 1}

The following deduction of Theorem 1 from the above lemmas is standard (cf. Section 6.5 of [10]), but we present it for the convenience of readers.

We begin with the Mergelyan theorem.

LEMMA 7. Let $K$ be a compact subset of $\mathbb{C}$ whose complement is connected. Then any continuous function $f(s)$ on $K$ which is analytic in the interior of $K$ is approximable uniformly on $K$ by polynomials of $s$.

Proof is given, for example, in [20].

Proof of Theorem 1. First suppose that functions $f_{l}(s), l=1, \ldots, n$, can be continued analytically to the whole of $D$. Denote by $\mathcal{G}$ the set of all $\left(g_{1}, \ldots, g_{n}\right) \in H^{n}(D)$ such that

$$
\sup _{1 \leq l \leq n} \sup _{s \in K_{l}}\left|g_{l}(s)-f_{l}(s)\right|<\frac{\varepsilon}{4} .
$$

Let $P_{n}$ and $P$ be probability measures defined on $(S, \mathcal{B}(S))$. It is well known (see [4], Theorem 2.1) that $P_{n}$ converges weakly to $P$ as $n \rightarrow \infty$ if and only if

$$
\liminf _{n \rightarrow \infty} P_{n}(G) \geq P(G)
$$

for all open sets $G$. 
The set $\mathcal{G}$ is open, and, by Lemma 1 , the measure $P_{T}$ converges weakly to $P_{L}$ as $T \rightarrow \infty$. Therefore, using the above property of the weak convergence of probability measures and Lemma 6 , we obtain

(19) $\liminf _{T \rightarrow \infty} \nu_{T}\left(\sup _{1 \leq l \leq n} \sup _{s \in K_{l}}\left|L\left(\lambda_{l}, \alpha_{l}, s+i \tau\right)-f_{l}(s)\right|<\frac{\varepsilon}{4}\right)=P_{L}(\mathcal{G})>0$.

Now let the functions $f_{l}(s), l=1, \ldots, n$, be the same as in the statement of Theorem 1. By Lemma 7 there exist polynomials $p_{l}(s), l=1, \ldots, n$, such that

$$
\sup _{1 \leq l \leq n} \sup _{s \in K_{l}}\left|p_{l}(s)-f_{l}(s)\right|<\frac{\varepsilon}{2} .
$$

By the first part of the proof we have that

$$
\liminf _{T \rightarrow \infty} \nu_{T}\left(\sup _{1 \leq l \leq n} \sup _{s \in K_{l}}\left|L\left(\lambda_{l}, \alpha_{l}, s+i \tau\right)-p_{l}(s)\right|<\frac{\varepsilon}{2}\right)>0 .
$$

Obviously, for $l=1, \ldots, n$

$$
\sup _{s \in K_{l}}\left|L\left(\lambda_{l}, \alpha_{l}, s+i \tau\right)\right| \leq \sup _{s \in K_{l}}\left|L\left(\lambda_{l}, \alpha_{l}, s+i \tau\right)-p_{l}(s)\right|+\sup _{s \in K_{l}}\left|f_{l}(s)-p_{l}(s)\right| .
$$

Therefore by (20) it is easily seen that

$$
\begin{aligned}
& \left\{\tau: \sup _{1 \leq l \leq n} \sup _{s \in K_{l}}\left|L\left(\lambda_{l}, \alpha_{l}, s+i \tau\right)-f_{l}(s)\right|<\varepsilon\right\} \\
& \supseteq\left\{\tau: \sup _{1 \leq l \leq n} \sup _{s \in K_{l}}\left|L\left(\lambda_{l}, \alpha_{l}, s+i \tau\right)-p_{l}(s)\right|<\frac{\varepsilon}{2}\right\} .
\end{aligned}
$$

This and (21) yield the assertion of Theorem 1.

Now we discuss briefly the case that $1, \lambda_{1}, \ldots, \lambda_{n}$ are linearly independent over $\mathbb{Q}$. Then the sequence

$$
\left\{\left(\lambda_{1} m, \ldots, \lambda_{n} m\right), m \in \mathbb{N}\right\}
$$

is uniformly distributed mod 1 in $\mathbb{R}^{n}$ (see Kuipers-Niederreiter [17], Section 1.6, Example 6.1), hence the set

$$
N_{\varepsilon}=\left\{m \in \mathbb{N}:\left(\lambda_{1} m, \ldots, \lambda_{n} m\right) \in(-\varepsilon, \varepsilon)^{n} \bmod 1\right\}
$$


has the positive density $(2 \varepsilon)^{n}$. From (11) we have

$$
\sum_{m \in N_{\varepsilon}}\left|\sum_{l=1}^{n} e^{2 \pi i \lambda_{l} m} \varrho_{l}(\log m)\right|<\infty,
$$

which suggests that

$$
\sum_{m \in N_{\varepsilon}}\left|\sum_{l=1}^{n} \varrho_{l}(\log m)\right|<\infty
$$

might be also true. If (23) would be true, then using Lemmas 4 and 5 we obtain

$$
\sum_{l=1}^{n} \varrho_{l}(z) \equiv 0
$$

for any $z \in \mathbb{C}$. We could prove

$$
-\varrho_{1}(z)+\sum_{l=2}^{n} \varrho_{l}(z) \equiv 0
$$

in the same way, hence $\varrho_{1}(z) \equiv 0$, and similarly $\varrho_{l}(z) \equiv 0, l=2,3, \ldots, n$. From this fact we could deduce the joint universality theorem in this case. If we could prove the above conclusion $\varrho_{l}(z) \equiv 0$ not only from $(23)$, but also from (22), then this argument would be complete.

\section{Proof of Theorem 2}

It is sufficient to give a sketch, because the proof is a direct generalization of that in [6]. Define the mapping $h: \mathbb{R} \rightarrow \mathbb{C}^{N n}$ by

$$
\begin{aligned}
h(t)= & \left(L\left(\lambda_{1}, \alpha_{1}, \sigma+i t\right), \ldots, L\left(\lambda_{n}, \alpha_{n}, \sigma+i t\right),\right. \\
& L^{\prime}\left(\lambda_{1}, \alpha_{1}, \sigma+i t\right), \ldots, L^{\prime}\left(\lambda_{n}, \alpha_{n}, \sigma+i t\right), \ldots, \\
& \left.L^{(N-1)}\left(\lambda_{1}, \alpha_{1}, \sigma+i t\right), \ldots, L^{(N-1)}\left(\lambda_{n}, \alpha_{n}, \sigma+i t\right)\right) .
\end{aligned}
$$

For any $\varepsilon>0$ and any $s_{\nu l} \in \mathbb{C}(0 \leq \nu \leq N-1,1 \leq l \leq n)$, we can find $\tau \in \mathbb{R}$ such that

$$
\left|L^{(\nu)}\left(\lambda_{l}, \alpha_{l}, \sigma+i \tau\right)-s_{\nu l}\right|<\varepsilon \quad(0 \leq \nu \leq N-1,1 \leq l \leq n) .
$$

This can be shown by the same way as in Lemma 3 of [6], by taking the polynomial

$$
p_{l N}(s)=\sum_{\nu=0}^{N-1} \frac{s_{\nu l} s^{\nu}}{\nu !} \quad(1 \leq l \leq n)
$$


and applying Theorem 1 . Hence the image of $\mathbb{R}$ by the mapping $h$ is dense in $\mathbb{C}^{N n}$. From this, similarly to [6] (or Section 6.6 of [10]), we can deduce the conclusion of Theorem 2 .

\section{REFERENCES}

[1] M. Amou and M. Katsurada, Differential transcendence of a class of generalized Dirichlet series, in preparation.

[2] B. Bagchi, The statistical behaviour and universality properties of the Riemann zeta-function and other allied Dirichlet series, Ph. D. Thesis, Calcutta, Indian Statistical Institute, 1981.

[3] $\longrightarrow$ Joint universality theorem for Dirichlet L-functions, Math. Zeitschrift, 181 (1982), 319-334.

[4] P. Billingsley, Convergence of Probability Measures, John Wiley, New York, 1968.

[5] R. Garunkštis, The universality theorem with weight for the Lerch zeta-function, In: Analytic and Probabilistic Methods in Number Theory, New Trends in Probability and Statistics, Vol.4, A. Laurinčikas et al. (Eds.), VSP/TEV, 1997, pp. 59-67.

[6] R. Garunkštis and A. Laurinčikas, On one Hilbert's problem for the Lerch zetafunction, Publ. Inst. Math. (Beograd) (N.S.), 65 (79) (1999), 63-68.

[7] S.M. Gonek, Analytic properties of zeta and L-functions, Ph. D. Thesis, University of Michigan, 1979.

[8] A. Laurinčikas, On the zeros of linear forms of Dirichlet series, Liet. Matem. Rink., 26(3) (1986), 468-477 (in Russian) = Lith. Math. J., 26(3) (1986), 244-251.

[9] Application of probability methods in the theory of Dirichlet series, Thesis Doctor of Sciences, Vilnius, 1989 (in Russian).

[10] L Limit Theorems for the Riemann Zeta-Function, Kluwer Academic Publishers, Dordrecht/ Boston/ London, 1996.

[11] — On limit distribution of the Lerch zeta-function, In: Analytic and Probabilistic Methods in Number Theory, New Trends in Probability and Statistics, Vol.4, A. Laurinčikas et al.(Eds.), VSP/TEV, 1997, pp.135-148.

[12] —, The universality of the Lerch zeta-function, Liet. Matem. Rink., 37(3) (1997), 367-375 (in Russian) = Lith. Math. J., 37(3) (1997), 275-280.

[13] - On the Lerch zeta-function with rational parameters, Liet. Matem. Rink., 38(1) (1998), 113-124 (in Russian) = Lith. Math. J., 38(1) (1998), 89-97.

[14] On the zeros of linear combinations of the Matsumoto zeta-functions, Liet. Matem. Rink., 38(2) (1998), 185-204 (in Russian) = Lith. Math. J., 38(2) (1998), $144-159$.

[15] A. Laurinčikas and K. Matsumoto, Joint value-distribution theorems on Lerch zeta-functions, Liet. Matem. Rink., 38(3) (1998), 312-326 = Lith. Math. J., 38(3) (1998), 238-249.

[16] M. Lerch, Note sur la fonction $K(w, x, s)=\sum_{n \geq 0} \exp \{2 \pi i n x\}(n+w)^{-s}$, Acta Math., 11 (1887), 19-24. 
[17] L. Kuipers and H. Niederreiter, Uniform Distribution of Sequences, John Wiley\& Sons, New York/London/Sydney/Toronto, 1974.

[18] S.M. Voronin, On the functional independence of Dirichlet L-functions, Acta Arith., 27 (1975), 493-503 (in Russian).

[19] S.M. Voronin, Analytic properties of generating Dirichlet functions of arithmetical objects, Thesis Doctor phys.-matem. nauk, Moscow, 1977 (in Russian).

[20] J.L. Walsh, Interpolation and Approximation by Rational Functions in the Complex Domain, Amer. Math. Soc. Coll. Publ., 20 (1960).

\author{
Antanas Laurinčikas \\ Department of Mathematics \\ Vilnius University \\ Naugarduko, 24 \\ 2006 Vilnius \\ Lithuania \\ antanas.laurincikas@maf .vu.lt \\ Kohji Matsumoto \\ Graduate School of Mathematics \\ Nagoya University \\ Chikusa-ku, Nagoya, 464-8602 \\ Japan \\ kohjimat@math.nagoya-u.ac.jp
}

